\title{
A Heuristic Particle Swarm Optimization for Cutting Stock Problem Based on Cutting Pattern
}

\author{
Xianjun Shen ${ }^{1,2}$, Yuanxiang $\mathrm{Li}^{2}$, Jincai Yang ${ }^{1}$, and $\mathrm{Li} \mathrm{Yu}^{2}$ \\ ${ }^{1}$ Department of Computer Science \\ Central China Normal University, Wuhan 430079, China \\ ${ }^{2}$ State Key Lab of Software Engineering \\ Wuhan University, Wuhan 430072, China \\ xjshen@mail.ccnu.edu.cn
}

\begin{abstract}
A heuristic particle swarm optimization (HPSO) is proposed as a solution to one-dimensional cutting stock problem (1D-CSP), which incorporate genetic operators into particle swarm optimization (PSO). In this paper, a heuristic strategy that is based on the results of analysis of the optimal cutting pattern of particles with successful search processes is described, which process a global optimization problem of the cutting-stock as a sequential optimization problem by multiple stages. During every sequential stage, the best cutting pattern for the current situation is researched and processed. This strategy is repeated until all the required stocks have been generated. The simulation results prove the effectiveness of the proposed methodology.
\end{abstract}

Keywords: Heuristic particle swarm optimization, One-dimensional cutting stock problem, Genetic operators.

\section{Introduction}

The one-dimensional cutting stock problem (1D-CSP) is one of the representative combinatorial optimization problems, which arises in many industries [1. This paper proposes a heuristic particle swarm optimization (HPSO) incorporating genetic algorithm (GA). The main idea of HPSO is to process a global optimization problem of the cutting-stock as a sequential optimization problem by multiple stages. During every sequential stage, the best cutting pattern for the current situation is researched and processed. This stage processing is repeated until all the required stocks have been generated. The experiment result shows that the HPSO can obtain satisfying effect.

\section{One-Dimensional Cutting Stock Problem}

In the pattern-oriented approach, at first, order lengths are combined into cutting patterns, however, it is impractical to consider all feasible cutting patterns, and 
we would try to find a set of $n$ better cutting patterns yielding small deviations from the order demands. In a succeeding step, the frequencies are determined that are necessary to satisfy the order demands.

One of the most important costs for 1D-CSP is the amount of residual pieces of processed stock, called trim loss, which are usually treated as waste product. Hence, the problem of minimizing the total trim loss (or the number of processed stock) is considered as the most important factor.

To define 1D-CSP, it is given a sufficient number of stocks, which have the same length $L$, and $m$ types of products with given lengths $\left(l_{1}, l_{2}, \ldots, l_{m}\right)$ and their demands $\left(d_{1}, d_{2}, \ldots, d_{m}\right)$. A cutting pattern is a combination of products cut from a stock. A cutting pattern is described as $p_{j}=\left(a_{1 j}, a_{2 j}, \ldots, a_{m j}\right)$, where $a_{i j}$ is the number of product $i$ cut from one stock roll. It is suppose that have found $\mathrm{n}$ type optimal cutting patterns, the first cutting pattern is cut the quantity of the $\mathrm{m}$-th type product is $a_{m 1}$, and its the trim loss is $b_{1}$. The second cutting pattern is cut the quantity of the m-th type product is $a_{m 2}$, and its the trim loss of the second is $b_{2}$. Thus, the cutting pattern $n$ is cut the quantity of the m-th type product is $a_{m n}$, and its the trim loss is $b_{n}$ [2]. The mathematics model of one-dimensional cutting stock problem is formulated as follows:

$$
\mathbf{A}=\left[\begin{array}{cccc}
a_{11} & a_{12} & \ldots & a_{1 n} \\
a_{21} & a_{22} & \ldots & a_{2 n} \\
\ldots & \ldots & \ldots & \ldots \\
a_{m 1} & a_{m 2} & \ldots & a_{m n}
\end{array}\right]
$$

The number of stock that use $\mathrm{n}$ type different cutting pattern is as follow:

$$
X=\left(x_{1}, x_{2}, \ldots, x_{n}\right)^{T}
$$

The trim loss of cutting pattern $p_{j}$ is as follows.

$$
b_{j}=L-\left(a_{1 j} l_{1}+a_{2 j} l_{2}+\ldots+a_{m j} l_{m}\right) \quad j=1,2, \ldots, n
$$

Where $a_{m j}$ is the number of m-th type product that use cutting pattern $p_{j}$. Now the object function of the pattern-oriented approach is formulated as follows.

$$
f(x)=\min \left(\sum_{j=1}^{n} b_{j} x_{j}\right)
$$

The last stock has the maximum remainder and can in general be used further, thus it is not considered as waste. The summation of trim loss in one-dimensional cutting stock problem should be subtracted the remainder of the last stock.

$$
f(x)=\min \left(\sum_{j=1}^{n} b_{j} x_{j}-L^{\prime}\right)(1 \leq i \leq k)
$$

Where $L^{\prime}$ denotes indicate the longest remainder of a cutting plan which cumulate consecutive residual lengths in one stock which could be used later. 


\section{Heuristic Particle Swarm Optimization}

PSO is a swarm intelligence optimization algorithm. The information exchange takes place only among the particle's own experience and the experience of the best particle in the swarm. HPSO model is still based on PSO mechanism, but use genetic operator as the updating operator. The best position of each particle would update by crossover operator and mutation operator.

In heuristic particle swarm optimization, each potential optimal cutting plan, called a particle, the current position of the particle is denoted $x_{i}^{t}$. The particles have memory and each particle keep track of its previous best position (denoted $x b e s t_{i}^{t}$ ) and its corresponding fitness. The particle with greatest fitness is called the global best and its position is called the global best position (gbest). Each particle towards it's $x_{b e s t}^{t}$ and the gbest position. The current number of iteration generation is represented by the symbol $t$.

The main steps in HPSO Process are described as follows:

Step 1. Initialize the swarm randomly.

Step 2. For each particle of swarm, Choose $x_{i}^{t}$ and $x b e s t_{i}^{t}$, and generate $x_{i}^{t}$ by crossover operator.

Step 3. For each particle of swarm, Choose $x_{i}^{\prime}{ }^{t}$ and $x_{g b e s t}$, and generate $x_{i}^{\prime \prime} t$ by crossover operator.

Step 4. For each particle of swarm, Choose $x_{i}^{\prime \prime} t$, and generate $x_{i}^{t+1}$ by mutation operator.

Step 5. Calculate the fitness each particle.

Step 6. Update the best position of each particle so far.

Step 7. Update the global best position of the whole swarm so far.

Step 8. Repeat Step 2 to Step 6, Choose the best cutting pattern for the current situation.

Step 9. Process cutting stocks by the best cutting pattern, subtract the quantities of the every type pieces that have been cut, and then update each particle of swarm.

Step 10. If all the required stocks have been not generated, go to step 2.

Step 11. Output the final results of HPSO.

\section{Experiments Results}

One of the most important costs for 1D-CSP is the amount of residual pieces of processed stock, called trim loss. The purpose of HPSO is that search a cutting plan that has the least trim loss The benchmark instance was given in [3. The optimal cutting plan was given in 3] by a hybrid genetic algorithm which the longest remainder of stock is $2746 \mathrm{~mm}$. The total trim loss of other stocks is $2411 \mathrm{~mm}$.

According to the results in Table.1, HPSO has attained a satisfying optimal cutting plan. The optimal cutting plan is given in table 1 which the longest remainder of stock is $133 \mathrm{~mm}$. The total trim loss of other stocks is $163 \mathrm{~mm}$. The average availability ratio of other stocks is $99.86 \%$. The result is satisfactory that overall stocks needed by all cuts are minimized. 
Table 1. Solution of instance with HPSO

\begin{tabular}{|c|c|c|c|c|c|}
\hline No. & $\begin{array}{l}\text { Stock } \\
\text { length }\end{array}$ & $\begin{array}{c}\text { Pieces length } \\
\text { (Pieces amount) }\end{array}$ & $\begin{array}{c}\text { Trim } \\
\text { loss }\end{array}$ & $\begin{array}{l}\text { Availability } \\
\text { ratio }\end{array}$ & $\begin{array}{l}\text { Pattern } \\
\text { number }\end{array}$ \\
\hline 1 & \multirow{10}{*}{6000} & $1694(2) 1687925$ & 0 & 100 & 2 \\
\hline 2 & & $885(3) 855(2) 828807$ & 0 & 100 & 2 \\
\hline 3 & & $1464978925889(2) 855$ & 0 & 100 & 2 \\
\hline 4 & & 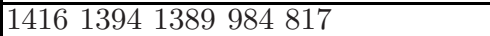 & 0 & 100 & 1 \\
\hline 5 & & \begin{tabular}{|llllll}
984 & 925 & 828 & $817(3)$ & 811
\end{tabular} & 1 & 99.99 & 1 \\
\hline 6 & & 213714941387978 & 4 & 99.93 & 1 \\
\hline 7 & & 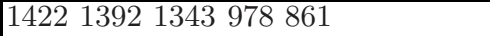 & 4 & 99.93 & 1 \\
\hline 8 & & \begin{tabular}{|l}
$1094(3) 978925811$ \\
\end{tabular} & 4 & 99.93 & 1 \\
\hline 9 & & \begin{tabular}{|llll}
1494 & 1422 & 1419 & $828(2)$
\end{tabular} & 9 & 99.85 & 1 \\
\hline 10 & & \begin{tabular}{|lllll}
2144 & 1446 & 1416 & 861
\end{tabular} & 133 & 97.78 & 1 \\
\hline 11 & \multirow{10}{*}{8000} & 214412961167889885811808 & 0 & 100 & 2 \\
\hline 12 & & \begin{tabular}{|lllll}
1541 & 1464 & $1167(2)$ & $925(2)$ & 811 \\
\end{tabular} & 0 & 100 & 1 \\
\hline 13 & & \begin{tabular}{|llllllll}
1389 & 1167 & 1094 & 984 & 889 & 861 & $808(2)$
\end{tabular} & 0 & 100 & 1 \\
\hline 14 & & $21371167(2) 1107808807(2)$ & 0 & 100 & 1 \\
\hline 15 & & \begin{tabular}{|llllll}
1422 & 1392 & 984 & $861(3)$ & 811 & 808
\end{tabular} & 0 & 100 & 1 \\
\hline 16 & & \begin{tabular}{|llll}
$1494(2)$ & 1416 & $1392(2)$ & 811
\end{tabular} & 1 & 99.99 & 1 \\
\hline 17 & & \begin{tabular}{|lllllll}
2137 & 1426 & 1416 & 1400 & 811 & 808
\end{tabular} & 2 & 99.97 & 1 \\
\hline 18 & & 21371676146414191296 & 8 & 99.90 & 1 \\
\hline 19 & & $21441676 \quad 142213891337$ & 32 & 99.60 & 1 \\
\hline 20 & & $138911671107 \quad 861(3) 828(2)$ & 98 & 98.78 & 1 \\
\hline 21 & \multirow{2}{*}{9000} & 1081(4) 1034(2) 984817807 & 0 & 100 & 4 \\
\hline 22 & & \begin{tabular}{|lllllll}
$978(3)$ & 925 & 906 & 889 & $855(2)$ & 828 & 808 \\
\end{tabular} & 0 & 100 & 1 \\
\hline
\end{tabular}

\section{Conclusion}

This paper presents a heuristic particle swarm optimization algorithm to solve a one-dimensional cutting stock problem, which merges crossover operator, mutation operator, and heuristic strategy based on best cutting pattern to explore the best cutting plan. The simulation result shows the proposed heuristic algorithm is applied successfully to solve 1D-CSP.

\section{References}

1. Gradišar, M., Trkman, P.: A combined approach to the solution to the general one-dimensional cutting stock problem. Computers and Operations Research 32(7) (2005) 1793-1807

2. Jia, Z., Yin, G., Hu, X., Shu, B.: Optimization for one-dimensional cutting stock problem based on genetic. Journal of Xian'an Jiaotong University 36 (2002) 967-970

3. Peiyong, L.: Optimization for variable inventory of one-dimensional cutting stock. Mechanical Science and Technology 22(supplement) (2002) 80-86 